\title{
Refractive index profile modelling of dielectric inhomogeneous coatings using effective medium theories
}

\author{
Vesna Janicki ${ }^{*}$, Jordi Sancho-Parramon, Hrvoje Zorc \\ Ruđer Bošković Institute, Bijenička c. 54, 10000 Zagreb, Croatia
}

\begin{abstract}
The coatings having refractive index changing with the thickness present interesting optical performance, improved mechanical properties and smaller light scattering in comparison with classical multilayer stacks. Lot of theoretical work and experimental advances have been done for designing and production of mixture layers with such particular performances. The effective refractive index of the mixture coatings can be calculated by the use of effective medium theories. The refractive index profile characterization of inhomogeneous films that are mixtures of $\mathrm{SiO}_{2}$ and $\mathrm{Nb}_{2} \mathrm{O}_{5}$ is presented. The composition is linearly changed through the thickness of the layers. Exsitu spectrophotometric measurements, i.e. reflectance and transmittance at different incidence angles, are used for the precise characterization of the refractive index profiles. Linear, Maxwell-Garnet, Bruggeman and Lorentz-Lorenz effective medium theories are applied and quality and differences of the results are studied and analyzed. It is shown that the Lorentz-Lorenz model is the most appropriate for the given mixture, suggesting components are well mixed and there are no separated phases.
\end{abstract}

\footnotetext{
*Corresponding author. Tel. + 3851 4571247, fax: + 3851 4680104, e-mail: janicki@irb.hr
} 
Keywords: composite thin films, effective medium theory, optical coatings, optical characterization

\section{Introduction}

Progress in computer capabilities and advances in coatings process control has enabled design and deposition of gradient refractive index and rugate films with increasing complexity and precision. In this kind of coatings the variation of refractive index (n) varies through the depth of the film continuously, in contrast to the classical coatings with abrupt changes of refractive index. The coatings having smooth transition in refractive index, compared with classical quarter wave stacks, show better mechanical resistance [1,2], higher laser induced damage threshold [3] and less scattering losses, maintaining comparable or even better optical properties [4,5]. The variation of refractive index is obtained by variation of the composition through the thickness of the film. The standard techniques for deposition of such mixture coatings are sol-gel methods [6], changing the composition of the compounds by controlling the composition of reactive gas as in chemical vapour deposition [7], and co-deposition as in physical vapour deposition [8]. Calculation of optical constants of mixtures is important in design to predict the performance of the coating accurately. It is also important in analysis, i.e. optical characterisation, where model obtained by fitting of experimental spectra is compared with the targeted refractive index profile that was aimed to be deposited. Analysis of the differences between the two is crucial in detecting the errors of the deposition process and can be used for improvement of the manufacturing process. 


\section{Theory}

Effective medium theories relate optical properties of the mixtures with their composition. The effective refractive index of a mixture is calculated from the refractive indices and volume fractions of the composing materials. The most frequently used and most successful theories are Maxwell-Garnett [9] (MG), Bruggeman [10] (BG) and Lorentz-Lorenz [11] (LL). The first two assume that the mixing materials are in separated phases. Typical dimensions of the constituent particles are supposed to be much smaller than the wavelength of the interacting radiation, but at the same time large enough to present their own electromagnetic behaviour. MG considers the mixture that has separated a two-material grain structure where particles of the first material are dispersed in the continuous host of the second material. On the other hand, BG assumes an aggregate structure being a space-filling random mixture of two material phases. In the limit of small volume fractions $\left(f_{v}\right)$ the predictions of the two theories approach to each other. It is shown that in the case of higher filling factors, i.e. when volume fraction of one material is comparable to the volume fraction of another, the BG is valid up to the smaller particle radius than for MG [12]. LL model is based on the ClausiusMossoti equation and takes an average of molecular polarisability of the components. In this case no phase separation is considered. Another possibility is to assume linear variation of refractive index with volume fractions of constituents (LIN).

Finally, depending on the level at which materials are mixed, one effective medium theory will be more appropriate than the other and will give more accurate prediction of the optical properties of the mixture. For example, in the case of $\mathrm{SiO}_{2}-\mathrm{TiO}_{2}$ amorphous mixture it has been shown experimentally $[13,14]$ that LL model is more appropriate than BG, although not all the studies are in accordance [15]. 
Layers with approximately linear gradient of refractive index (ramps) are the basic building elements of more complex hybrid [16] and good approximation for rugate coatings. They are deposited by linear variation in volume fraction $f_{v}$ of constituents, from some minimum to maximum. Due to the above mentioned reasons it is important to check how much the different effective medium theories are capable to represent the refractive index profile of the layers obtained by linear change in volume fraction of the materials. For this purpose samples with linear variation of volume fraction of $\mathrm{SiO}_{2}$ and $\mathrm{Nb}_{2} \mathrm{O}_{5}$ are prepared. Optical characterization from the measured reflectance $(\mathrm{R})$ and transmittance $(\mathrm{T})$ spectra is performed using MG, BG, LL and LIN effective medium theories. The quality of the fits to the experimental data are compared, also regarding the number of sublayers into each gradient was subdivided for the purpose of the calculations.

\section{Experimental details}

Layers of $\mathrm{SiO}_{2}-\mathrm{Nb}_{2} \mathrm{O}_{5}$ mixtures with composition changing linearly through the thickness of the film were deposited onto suprasil substrates by reactive electron beam evaporation co-deposition technique in a Leybold Syrus Pro 1100 deposition system. The chamber was equipped with two electron beam guns. The change in the composition is achieved by a continuous modification in deposition rates ( $\mathrm{r}$ ) of individual materials. The rate of deposition is controlled and measured by two quartz crystal monitors one for each material, next to the source. The deposition process is controlled by computer software enabling simultaneous automatic measurements and acquisition of parameters (deposition rates, pressures, temperature etc.) during the 
deposition. Volume fraction $f_{\mathrm{v} 1}$ of the material 1 in the mixture is related to the measured deposition rates of each material $r_{1}$ and $r_{2}$ by:

$$
\mathrm{f}_{\mathrm{v} 1}=\mathrm{r}_{1} /\left(\mathrm{r}_{1}+\mathrm{r}_{2}\right)
$$

The minimum and maximum deposition rates $\left(r_{m}\right.$ and $\left.r_{M}\right)$ of each material, and therefore minimum and maximum volume fractions $\left(\mathrm{f}_{\mathrm{vm}}\right.$ and $\left.\mathrm{f}_{\mathrm{vM}}\right)$, were restricted due to the fact that it was not technically possible to achieve stable and reproducible arbitrarily low deposition rates for the given materials. Thus, $r_{m}$ was set to $0.2 \mathrm{~nm} / \mathrm{s}$ and $r_{M}$ to $1.2 \mathrm{~nm} / \mathrm{s}$. The total rate of deposition, i.e. sum of the rates of the two materials, was kept constant during the process and set to $1.4 \mathrm{~nm} / \mathrm{s}$.

Three samples were deposited: one with content of niobia increasing from the substrate (Sample 1), another with content of niobia decreasing from the substrate (Sample 2) and the third with the content first increasing and then decreasing (Sample 3). The deposition rates vary continuously during the deposition, giving place to continuous change of the material composition. The thickness $d$ of the first two samples was approximately $220 \mathrm{~nm}$ and $560 \mathrm{~nm}$ for the third.

Prior to deposition of the samples substrate and pure material layers have been characterized and their refractive indices determined. Spectrophotometric measurements were performed with a Perkin Elmer Lambda 900 spectrophotometer. A VN-attachment allowing absolute measurement of reflectance (R) without moving the sample after transmittance $(\mathrm{T})$ measurement has been used. Reflectance and transmittance in the spectral range 400-900 nm were measured in steps of $2 \mathrm{~nm}$ at angle of incidence of 6 degrees and $\mathrm{s}$ and $\mathrm{p}$ polarizations $\mathrm{R}_{\mathrm{s}}, \mathrm{R}_{\mathrm{p}}, \mathrm{T}_{\mathrm{s}}$ and $\mathrm{T}_{\mathrm{p}}$ at 45 degrees.

\section{Optical characterization}


Optical characterization of the samples was done using thin film curve fitting software [17]. Fitting the experimental spectra allows determination of the optimal value of a set of parameters defining the sample. The variation of refractive index with thickness (inhomogeneity) of a ramp is taken into account by dividing it into a given number of homogeneous sublayers. For each ramp, all sublayers have the same thickness. Each sublayer has been modelled as a mixture of the two materials with refractive indices $n_{H}$ and $n_{L}$ and corresponding volume fractions $f_{H}$ and $f_{L}$, where $f_{H}+f_{L}=1$. Volume fraction of niobia in each layer is given by:

$$
f_{H-i}=f_{H-\text { start }}+\frac{f_{H-e n d}-f_{H-\text { start }}}{N_{\text {sub }}}\left(i-\frac{1}{2}\right) \text {. }
$$

Here $\mathrm{f}_{\mathrm{H}-\mathrm{i}}$ is volume fraction of niobia in the $\mathrm{i}$-th sublayer and $\mathrm{f}_{\mathrm{H} \text {-start }}$ and $\mathrm{f}_{\mathrm{H} \text {-end }}$ are niobia fractions at the beginning and the end of the ramp and $N_{s u b}$ is the number of sublayers. The effective dielectric function of the mixtures was calculated using different mixing formulas:

$$
\begin{array}{ll}
\text { MG } & \frac{\varepsilon_{\text {eff }}-\varepsilon_{H}}{\varepsilon_{e f f}+2 \varepsilon_{H}}=\left(1-f_{H}\right) \frac{\varepsilon_{L}-\varepsilon_{H}}{\varepsilon_{L}+2 \varepsilon_{H}} \\
\mathrm{BG} & f_{H} \frac{\varepsilon_{H}-\varepsilon_{e f f}}{\varepsilon_{H}+2 \varepsilon_{e f f}}+\left(1-f_{H}\right) \frac{\varepsilon_{\mathrm{L}}-\varepsilon_{e f f}}{\varepsilon_{L}+2 \varepsilon_{e f f}}=0 \\
\mathrm{LL} & \frac{\varepsilon_{\text {eff }}-1}{\varepsilon_{\text {eff }}+2}=f_{H} \frac{\varepsilon_{H}-1}{\varepsilon_{H}+2}+\boldsymbol{-} f_{H} \frac{-\varepsilon_{L}-1}{\varepsilon_{L}+2} \\
\mathrm{LIN} & \sqrt{\varepsilon_{\text {eff }}}=f_{H} \sqrt{\varepsilon_{H}}+\left(1-f_{H}\right) \sqrt{\varepsilon_{L}}
\end{array}
$$

where $\varepsilon_{\text {eff, }} \varepsilon_{\mathrm{H}}$ and $\varepsilon_{\mathrm{L}}$ are the effective dielectric functions of the mixture, high and low index material, respectively. The optical constants of the materials were used from data files determined from characterization of the layers of the pure materials, i.e. single layers of niobia and silica without gradients or mixing. In such a way the samples are represented by a limited set of parameters: $\mathrm{f}_{\mathrm{H}-\text { start }}, \mathrm{f}_{\mathrm{H}-\mathrm{end}}$ and thickness of the ramp and 
$\mathrm{N}_{\text {sub. }}$ Besides, putting limits to the parameters or fixing them, the optical characterization software enables establishing links between different parameters allowing the possibility of imposing the continuity of volume fractions at the interface of the two ramps as in Sample 3.

The quality of the optical characterization is evaluated by merit function (MF) which is minimized in the process of optimization. It is defined by:

$$
\chi^{2}=\frac{1}{N-m-1} \sum_{j=1}^{N_{s}} \sum_{k=1}^{N_{j}}\left(\frac{y_{k}^{j}-y^{j} \boldsymbol{q}_{k} ; P_{1}, \cdots, P_{m}}{\sigma_{k}^{j}}\right)^{2}
$$

where $N$ is the total number of experimental data points, $N_{s}$ is the number of measured spectra, each one containing $N_{j}$ experimental data points, $y_{k}^{j}$ represents measured values $(\mathrm{R}, \mathrm{Rs}, \mathrm{Rp}, \mathrm{Tp}, \mathrm{Rs}, \mathrm{Ts})$ at the wavelength $x_{k}$ with associated experimental error $\sigma_{k}^{j}$, $y_{j}\left(x_{k} ; P_{1}, \ldots, P_{m}\right)$ is the corresponding value calculated using standard thin film computation algorithms [18] and $P_{1}, \ldots, P_{m}$ are the $m$ parameters being optimized. The minimisation of the merit function is carried out using the Downhill-Simplex algorithm [19].

\section{Results}

The results presented here are obtained using the modelling described in the previous section and fitting simultaneously the experimental spectra $(R, R s, R p, T, T s$, Tp) all in the same wavelength range. The first sample, one with refractive index decreasing from the substrate, was taken to test the influence on the merit function of number of sublayers into which the model of was subdivided. The values of merit functions for 3, 5, 7, 13 and 25 layers applying LL and BG are shown in Table 1 and the corresponding refractive index profile and transmittance data fittings in Fig. 1.a. and Fig 
1.b. respectively. Comparison of refractive index profiles obtained by Maxwell-Garnet, Bruggeman, Lorentz-Lorenz and linear theory to model the same sample is presented in Fig. 2.a. and corresponding fittings and MF values in Fig. 2.b and Table 1. Optical characterization of all three samples is finally performed by dividing each ramp into two subramps, each consisting of 7 sublayers. This was done in order to take into account the possible nonlinearities of the deposition rates. Values of merit function obtained for the three samples when LL and BG were applied are presented in Table 2. The refractive index profiles, together with profiles calculated from deposition rates measured during deposition (using Equation 1 and then LL or BG), are shown in Fig. 3.a, 4.a. and 5.a. The transmittance data fittings are shown in Fig.3.b, 4.b and 5.b. The thickness of the profiles calculated from rates of deposition is normalized to those obtained from optical characterization. Recorded rates of deposition are shown to bee too much unstable to enable drawing conclusions from refractive index profiles calculated from the rates about convexity, concavity or number of ramps necessary to model the samples.

\section{Discussion}

From Table 1. it can be seen that division of the ramp into more than 5 sublayers doesn't contribute to the quality of the fit. For the given gradient of inhomogeneity, i.e. the change of refractive index 0.00268 per nanometre, the ramp is well approximated with homogeneous sublayers of $40 \mathrm{~nm}$ of thickness. This is important for computation purposes when a large number of ramps are involved in design or characterization. Even

so, in the continuation of this work a finer division was done. Of course, the number of sublayers doesn't change number of parameters for optimization. 
In Table 1 is also shown that LL gives merit function values 4-5 times lower than BG, MG or LIN. Merit functions of the last three models are comparable and from Fig. 2. a) one can see that also their refractive index profiles are quite similar to each other, while the one obtained by LL clearly differs. It shows concave shape, on the contrary of the others that are more linear. This can be related to the different nature of LL at respect to the other theories. As mentioned in Section 2, BG and MG model the mixture considering phase separation between components, in opposition to LL where components are so homogeneously mixed that it is not possible to speak about phase separation. The quantitative consequence of this conceptual difference is that BG and LL give place to an almost linear dependence of the refractive index with the compostion, while LL presents higher deviations from a linear dependence. Fig. 2. b) presents the differences of experimental and modelled (LL and BG) transmittances. The line that corresponds to the LL transmittance oscillates closer around zero value (dots at the Fig. 2. b)) indicating that this model describes optical properties better than BG model.

Splitting each ramp into two ramps with 7 sublayers each, introduced two more parameters for optimization: position of the junction of the two ramps and volume fraction of niobia in the point of junction. However, this is justified by significant decrease of merit function: from $1 / 3$ of the value down to 10 times lower value. From Table 2 it can be seen that LL again gives better MF values that BG (50\% better), except for the case of the first sample, where values are comparable. The nonlinearity of refractive index profile, in fact, originates from two sources: nonlinearity of deposition rates and nonlinear dependence of refractive index on volume fraction. From Fig. 3. it is possible to see that first sample gives the profile that is quite linear from its beginning to the end. Thus, when the first sample is presented by two ramps even BG is able to 
approach the concave profile. Value of its MF is lower comparing the model with only one ramp and at the same time comparable to the MF value of LL model with two ramps. LL model itself was not able to improve the quality of the fit significantly in the similar way as BG model, because the profile was concave already with only one ramp. The second sample (Fig. 4.a.) represents a convex profile. When modelled with one ramp BG was giving $25 \%$ better MF value than LL with its concave shape. The model with two ramps is convex for both effective medium theories used, representing convex profile originating from nonlinearity in rate of deposition. However, now LL gives $50 \%$ better MF value than BG, which corresponds to the nonlinearity caused by nonlinear relation of refractive index on volume fraction. Returning to the relation of the effective medium theories to the structure of the mixture, this would implicate that $\mathrm{SiO}_{2}$ and $\mathrm{Nb}_{2} \mathrm{O}_{5}$ are mixed at lower level than grains of the given materials. Indeed, analysis of FTIR spectra of the samples showed existence of Si-O-Nb bonds confirming that it is not possible to speak about different phases of these materials in the studied samples.

\section{Conclusions}

It is shown that the studied samples are well approximated by division into homogeneous sublayers with thickness of about $40 \mathrm{~nm}$. The division into more than 5 sublayers doesn't contribute to the quality of the fit to the measured spectra. Four effective medium theories were applied to the mixture of $\mathrm{SiO}_{2}$ and $\mathrm{Nb}_{2} \mathrm{O}_{5}$ : linear, Maxwell-Garnet, Bruggeman and Lorentz-Lorenz. The first three models gave similar results, but nonlinearities in refractive index profiles were the best described by the Lorentz-Lorenz model. Even in the case when nonlinearity was caused mainly by nonlinear rates of deposition resulting in convex shape, after splitting the ramp into two 
subramps Lorentz-Lorenz was able to give the fit of the quality comparable to the obtained by the use of Bruggeman model. The appropriateness of this model is confirmed also by the structural analysis of the mixture.

\section{Acknowledgments}

Vesna Janicki wishes to thank the Fraunhofer Society in Germany for a Fraunhofer Fellowship at IOF in Jena. The authors thank Heidi Haase for technical assistance. 


\section{References}

[1] D. Rats, D. Poitras, J. M. Soro, L. Martinu, J. von Stebut, Surf. Coat. Technol. 111 (1999) 220.

[2] M.-A. Raymond, S. Larouche, O. Zabeida, L. Martinu, J. E. Klemberg-Sapieha, Proceedings of the $44^{\text {th }}$ Annual Technical Conference of Society of Vacuum Coaters, Society of Vacuum Coaters, Albuquerque, N. Mexico, 2001 p.301.

[3] D. Ristau, H. Schink, F. Mittendorf, J. Ebert, and H. Welling., NIST Spec. Publ. 775 (1988) 414.

[4] V. Janicki, R, Leitel, S, Wilbrandt, O, Stenzel, D, Gäbler, N. Kaiser, Proc. SPIE 5963 (2005) 397.

[5] V. Janicki, M. Lappschies, B. Gortz, D. Ristau, U. Schallenberg, O. Stenzel, N. Kaiser, Proc. SPIE 5963 (2005) 5963.

[6] P. Prene, J. J. Priotton, L. Beavrain, P. Belleville, J. Sol-Gel Sci. Techn. 19 (2000) 533.

[7] H. Bartzsch, S. Lange, P. Frach, K. Goedicke, Surf. Coat. Technol. 180/181 (2004) 616.

[8] M. F. Ouellette, R. V. Lang, K. L. Yan, R. W. Bertram, R. S. Owles, D. Vincent, J. Vac. Sci. Technol. A, 9 (1991) 1188.

[9] J. C. Maxwell Garnett, Philos. T. Roy. Soc. A, 203 (1904) 385.

[10] D. A. Bruggeman, Ann. Phys. 24 (1935) 636.

[11] L. Lorenz, Ann. Phys. 11 (1880) 70.

[12] G. A. Niklasson, C.G. Grandqvist, O. Hunderi, Appl. Opt 20 (1981) 26.

[13] X. Wang, H. Masumoto, Y. Someno, T. Hirai, Thin Solid Films 338 (1999) 105. 
[14] S. Larouche, H. Szymanowski, J. E. Klemberg-Sapieha, L. Martinu, J. Vac. Sci. Technol. A, 22(4) (2004) 1200.

[15] J.-S. Chen, S. Chao, J.-S. Kao, H. Niu, C.-H. Chen, Appl. Opt. 35 (1996) 90.

[16] V. Janicki, S. Wilbrandt, O. Stenzel, D. Gäbler, N. Kaiser, A. Tikhonravov, M. Trubetskov, T. Amotchkina, J. Opt. A: Pure Appl. Opt. 7 (2005) L9.

[17] S. Bosch, J. Ferré-Borrull, J. Sancho-Parramon, Solid State Electron. 45 (2001) 703.

[18] P. H. Berning, Physics of thin films, Vol. 1, Academic Press, New York, 1963 p. 69.

[19] W. H. Press, S. A. Teukolsky, W. T. Vetterling, B. P. Flannery, Numerical Recipes in C, Cambridge University Press, New York, 1992. 


\begin{tabular}{|c|ccccc|}
\cline { 2 - 6 } \multicolumn{1}{c|}{} & 3 layers & 5layers & 7 layers & 13 layers & 25 layers \\
\hline LL & 0.2301 & 0.1390 & 0.1400 & 0.1404 & 0.1404 \\
BG & 0.4502 & 0.6244 & 0.6253 & 0.6198 & 0.6175 \\
MG & - & - & - & - & 0.8457 \\
LIN & - & - & - & - & 0.7844 \\
\hline
\end{tabular}

Table.1. Values of merit function obtained by use of different effective medium theories to the sample with refractive index decreasing from the substrate and division of the ramp into different number of sublayers.

\begin{tabular}{|cc|ccc|}
\cline { 3 - 5 } \multicolumn{1}{|c|}{} & Sample 1 & Sample 2 & Sample 3 \\
\hline LL & one ramp & 0.1404 & 1.0474 & 3.4891 \\
& two subramps & 0.0997 & 0.2602 & 0.2660 \\
\hline BG & one ramp & 0.6198 & 0.7337 & 3.6035 \\
& & & & \\
& two subramps & 0.0903 & 0.4109 & 0.4175 \\
\hline
\end{tabular}

Table.2. Values of merit function obtained by use of LL and BG before and after division of each ramp into two subramps. 

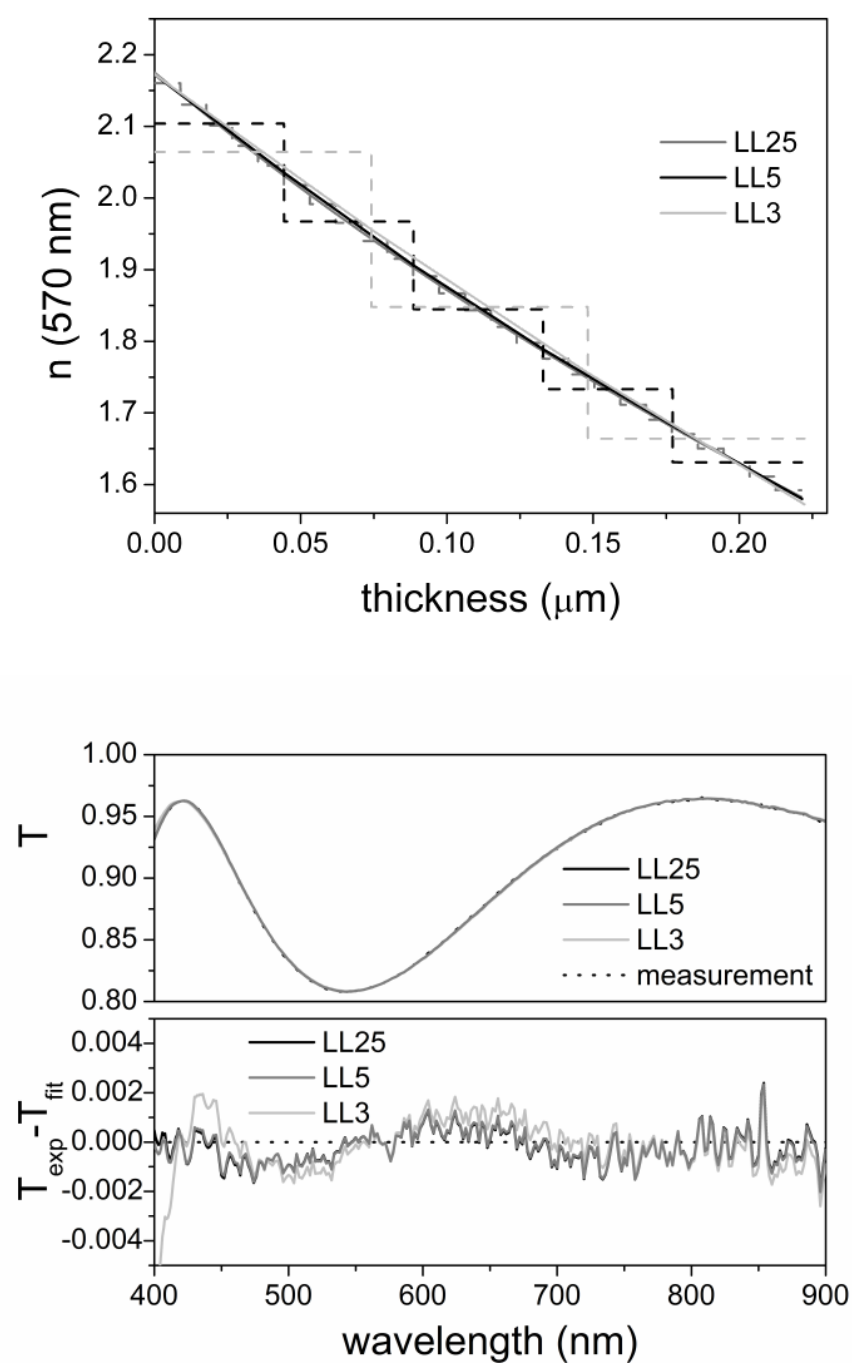

Fig. 1. Refractive index profiles obtained by division of the ramp of Sample 1 into 3, 5 and 25 sublayers and application of LL model (a). Dashed lines show actual division into sublayers and full lines present the slope obtained by this division, i.e. the profile of refractive index assuming a continuous variation of the volume fraction between $\mathrm{f}_{\mathrm{H} \text {-start }}$ and $\mathrm{f}_{\mathrm{H}-\mathrm{end}}$. The slope is shown to visualize the dependence of the refractive index with the volume fraction for each model. (b) Fitting of transmittance data at normal incidence and differences between fitting and experimental data for the different profiles. 

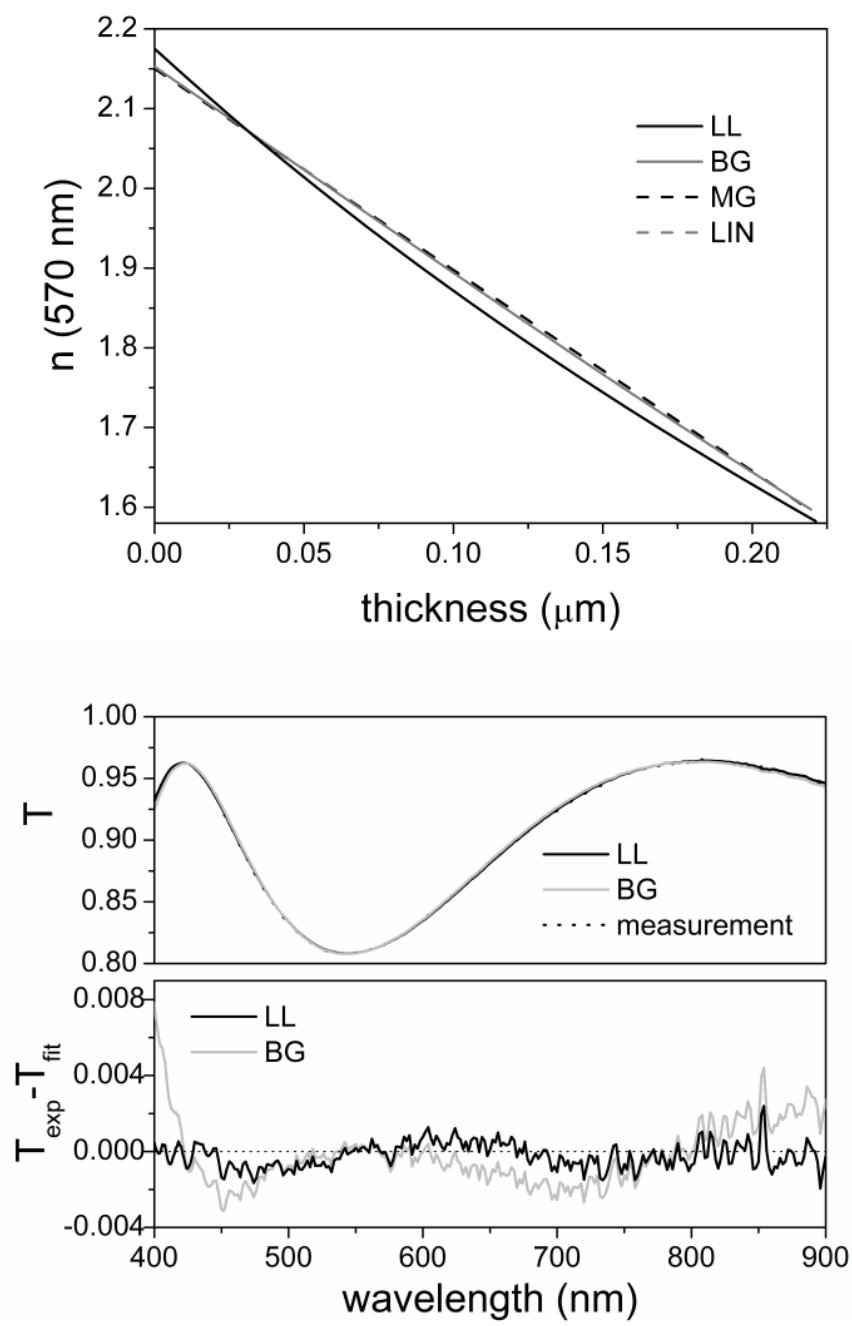

Fig. 2. Refractive index profiles obtained by division of the ramp of Sample 1 into 25 sublayers and application of different effective medium theories (a). Fitting of transmittance data at normal incidence and differences between fitting and experimental data for the different profiles (b). 

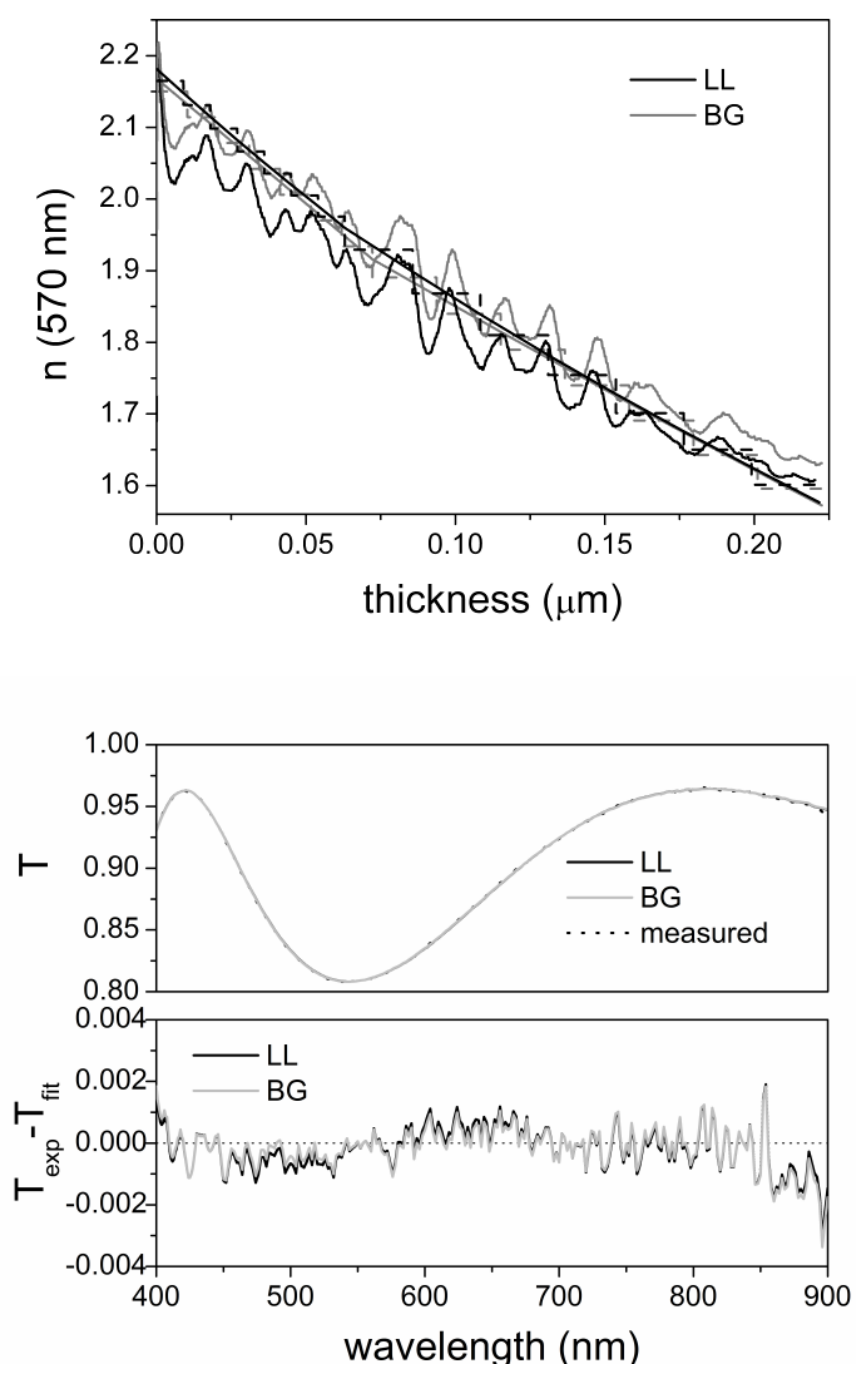

Fig. 3. Refractive index profiles obtained by division of the ramp of Sample 1 into 2 subramps, 13 layers each, and application of LL and BG (a). Dashed lines show actual division into sublayers, smooth full lines present the slope obtained by this division and rough lines are profiles calculated from rates of deposition. (b) Fitting of transmittance data at normal incidence and differences between fitting and experimental data for the different profiles. 

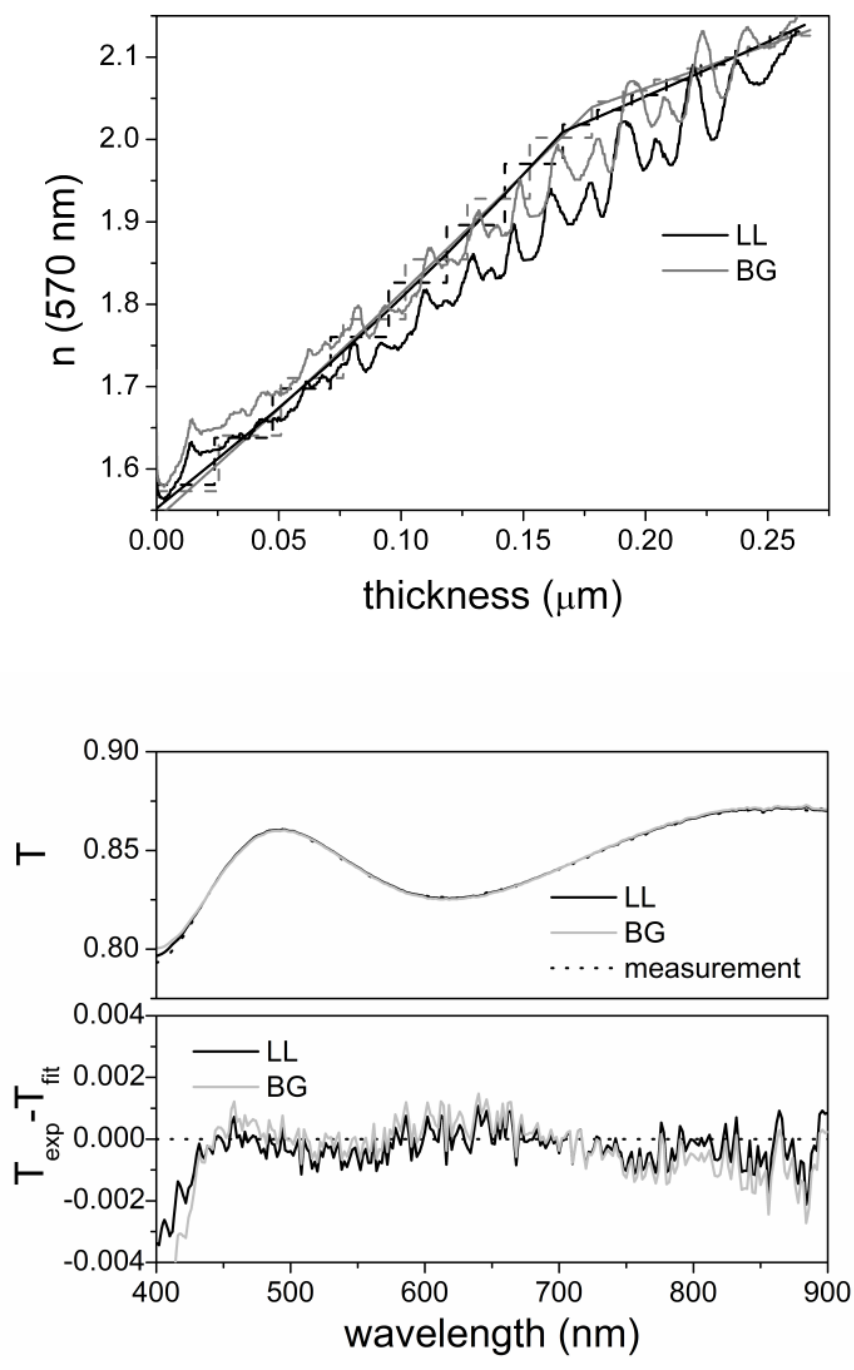

Fig. 4. Refractive index profiles obtained by division of the ramp of Sample 2 into 2 subramps, 13 layers each, and application of LL and BG (a). Dashed lines show actual division into sublayers, smooth full lines present the slope obtained by this division and rough lines are profiles calculated from rates of deposition. (b) Fitting of transmittance data at normal incidence and differences between fitting and experimental data for the different profiles. 

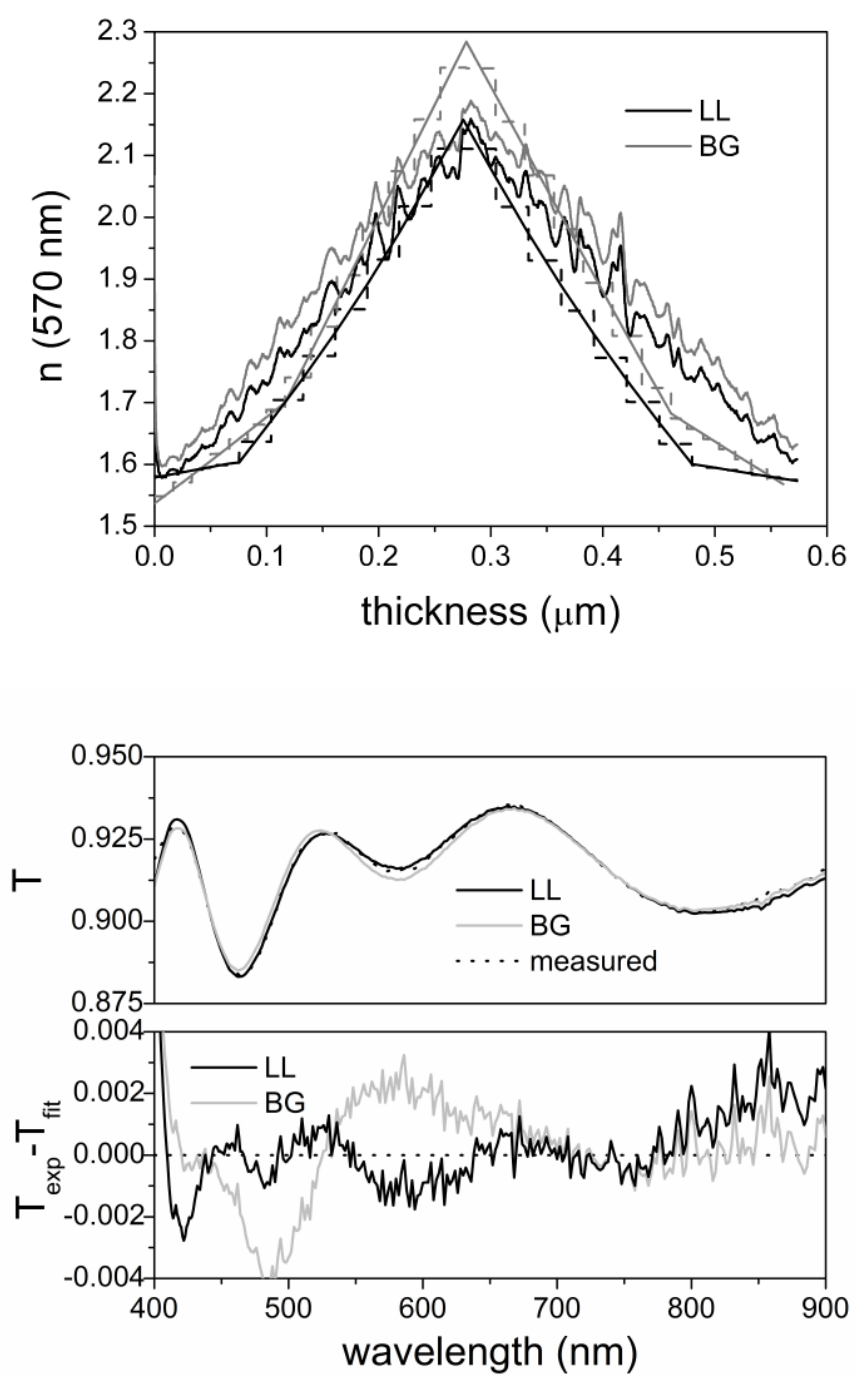

Fig. 5. Refractive index profiles obtained by division of the ramp of Sample 3 into 2 subramps, 13 layers each, and application of LL and BG (a). Dashed lines show actual division into sublayers, smooth full lines present the slope obtained by this division and rough lines are profiles calculated from rates of deposition. (b) Fitting of transmittance data at normal incidence and differences between fitting and experimental data for the different profiles. 